\title{
Global distributed energy systems
}

\author{
S. Lorente ${ }^{1} \&$ A. Bejan ${ }^{2}$ \\ ${ }^{1}$ Université de Toulouse, INSA, \\ Laboratoire Matériaux et Durabilité des Constructions, France \\ ${ }^{2}$ Duke University, \\ Department of Mechanical Engineering and Materials Science, USA
}

\begin{abstract}
In this paper the global design of energy production, distribution and sustainability is approached based on constructal theory. The global energy system is viewed as a conglomerate of flow systems morphing in time such that it provides greater and greater access to its currents (the constructal law). We show that from this principle follows the global design - the distribution of areas (users) allocated to nodes of power generation. Like the energy consumers themselves (e.g. vehicles, cities), the distribution of power generation on earth should be non uniform. Few large nodes should be interspaced with more numerous smaller nodes on a given territory. Together, this tapestry of areas and nodules serve the entire globe best. They also represent the best equilibrium between the flows of humanity and those of the environment. The paper builds an analogy between this design and the multi-scale design of the forest floor (the distribution of tree sizes) and the design of demography (the distribution of city sizes).
\end{abstract}

Keywords: constructal law, energy use, sustainability, distributed energy systems, Zipf distributions.

\section{Constructal law of generation of design}

The designs of animals show that the generation and use of motive power is distributed throughout the body. It is not centered in a single spot, nodule or organ. The animal muscle is a "tapestry" of patches served by two kinds of flow systems: (1) tissues that generate movement (contraction), and (2) vascularization that feeds, cleanses, and endows the tissue with the ability to sense and act. 
So perfect is the allocation of power generation to the networks for supply and distribution that the untrained eye sees the tissue as one or, at the most, as a complicated (multiscale) porous flow structure. The allocating of one flow system to the other flow system in the same confined space is the secret of the design. How are such designs made? How do they function?

These questions are interesting and highly promising when placed in the context of a sustainable energy future for our planet [1]. The inhabited surface of the earth is covered by the same two classes of flow systems:

(1) Nodules, large channels of power generation, embedded in

(2) Networks of supply and distribution.

Systems (1) and (2) are allocated to elemental areas, forming a patchwork that covers countries and continents. One example is the evolution of air mass transit: its map has history and memory. In time, new channels appear and old ones become thicker.

The flows of nature evolve in accordance with the constructal law: in time, they flow more and more easily, for greater access. (For a finite-size flow system to persist in time (to live), its configuration must change such that it provides greater and greater access to its currents $[2,3]$.) They attain this ever improving quality through the generation of configuration. Existing designs (literally, drawings) are replaced by new designs that flow more easily. Every flow system has its movie (animated cartoons), be it in nature or on our drawing boards. In this mental viewing we fit all the evolutionary scenarios of biology, the emergence of river basins and climate, and the evolution of technologies toward greater efficiency [1-9].

In evolution age matters, and it is good for flow performance. The river basin digs its channels better and better, and the channels stay in place. The channels have hierarchy: a few large channels flow in harmony with the many small channels. A sudden downpour is served well by the "memory" built into the old design.

Like the animal muscle, the patchwork of power generation, distribution, and use happens naturally. Unlike the animal muscle, which has spent millions of years in the factory of evolution, our energy systems evolve in front of our eyes. They morph while they grow. They produce more power, and they produce the power more efficiently.

Why do humans need power? For the same reason that animals need muscle power: to move mass on the earth's surface. Recent theoretical work on the constructal-law basis of animal locomotion [10] has shown that for all types of locomotion (running, flying, swimming), animal force is roughly equal to the body weight, and the minimum work that the body performs is proportional to the body weight times the distance traveled. The consumed food or fuel is "converted" into mass moved. Our cars, construction sites, and everything else that we do (our legacy) are the product of this. All the animals and all of us consume food and fuel, and the result is the shaping and reshaping (the mixing) of the earth's surface.

These are the questions that underpin the fundamental direction traced in this article. In addition to the importance of these fundamentals to the human design 
of the best possible sustainable and safe future, we see three more reasons for our confidence in this direction of inquiry:

First, the need to consider the whole is universal. Yet, most of the research on energy science and engineering is devoted to the "energy" side, that is, to more efficient and cleaner production (systems of type 1 above). The remainder of the effort is devoted to the "environment", that is, to the human flow networks that interact with the environment (systems of type 2 above). In constructal theory, energy (1) and the environment (2) are contemplated together, from the beginning, and from the smallest elemental area on which 1 and 2 come together to form a self-sustaining and long lasting tissue of flow systems.

Second, the widespread occurrence of distributed energy systems in nature is a very loud hint that the future of global energy design belongs to distributed systems. The future belongs to the vascularized [1]. In nature, distributed energy systems occur not only in animal design but also in inanimate flow systems such as river basins. Each sloped channel in a river basin is an optimal combination of (1) motive power (the slope, i.e., the driving gravitational potential); (2) distribution, use, dissipation (friction along the channel); and the allocation of 1 and 2 to the elemental territory bathed by the channel. The time arrow of evolution in natural flow systems points toward distributed energy systems.

Third, natural flow architectures have survived to this day because they have evolved into flow configurations that are the most efficient (they flow the easiest) under the constraints, for example, tree-shaped flows oriented point-toarea or area-to-point. They survived because they are free to adapt; that is, their configurations are malleable in spite of the constraints. Freedom is good for design [1]. We have and use freedom in the design of our own energy flow systems. Technology evolves by morphing, improving and spreading. There is no difference between natural flow architectures and the flow architectures constructed by humans and powered by the sources designed and positioned by humans on the landscape.

\section{Few large and many small, together}

With the constructal law, we have discovered this class of multi-scale designs on the landscape in three highly dissimilar domains. First was the distribution of city sizes and numbers on a continent (Fig. 1), where we predicted the observed Zipf-type distribution of city sizes vs. city ranks [11] (Fig. 1). The prediction followed from the observation that on every area element of the territory the number of those working on the area (the agriculturers) must be proportional to the number of the city dwellers, and both numbers must be proportional to the area allocated to them. This construction rule is illustrated in Fig. 1, and it generates the multi-scale distribution of demography that is correlated empirically as a straight line on the log-log field. The line moves upward, parallel to itself, because the evolution of technology: each area element sustains more people in time, on the land and in its allocated human settlement. 


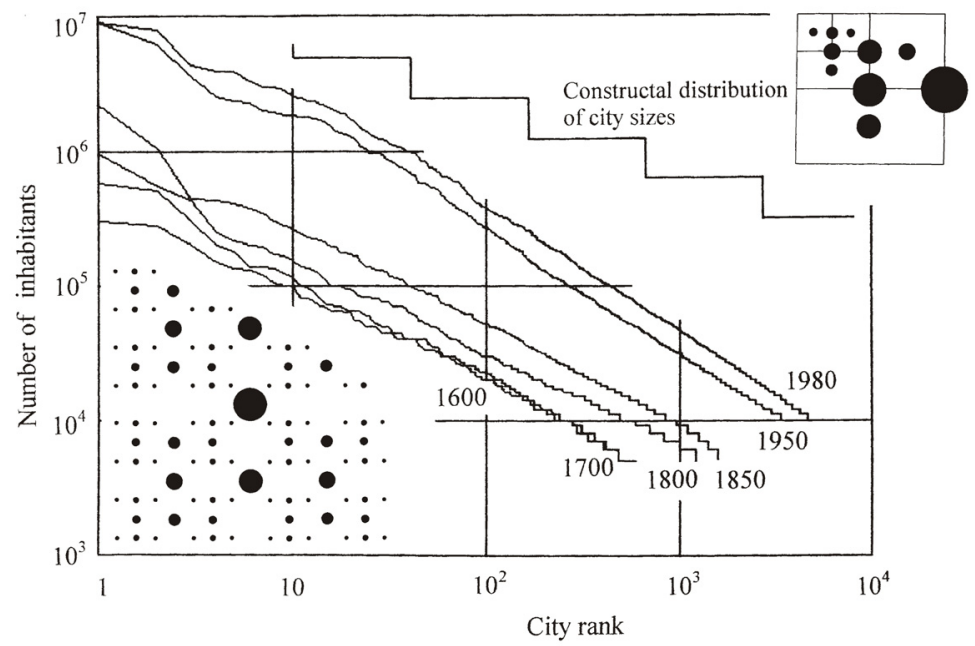

Figure 1: City sizes versus city rank in Europe during 1600-1980, and the design predicted with the constructal law [11].

The second example was the distribution of fuel use for heating [1]. Humanity needs heating all over the globe, and for this reason the burning of fuel occurs all over the globe. Key is the observation that all the generated heat (the used and the unused) is eventually discharged into the environment. The challenge is to channel most of this heat through our homes and enterprises before discharging it into the environment.

The challenge is to place humans and enterprises in the right places on the landscape, as optimally positioned interceptors. When this tapestry of interceptors of heat is designed from principle, two major objectives are achieved simultaneously:

- The heating needs of humanity are met by burning minimum fuel.

- The total heating dumped into the environment is the smallest that it can be.

We illustrated this by considering the hot-water needs of individuals spread uniformly on the landscape (Fig. 2). The area allocated to each has the fixed length scale d. Heat is being lost for two reasons: heat leaks from the furnaces represented by dots in Fig. 2 [six dots in (a), and one central dot in (b) and (c)], and heat leaks from the hot-water pipes shown as lines in Figs. 2(b) and (c). We found that when the individual need for hot water is small enough, the design with least heat loss per user is the individual design (a), in which each user relies on one heater, without pipes for hot water distribution. As the amount of hot water used by each individual increases, more economical is to use a central heater with radial lines of distribution to an optimal-size cluster of users (b). As the individual hot-water need increases with the evolution of the standard of living, even more economical are the dendritic clusters (c), which are larger and 


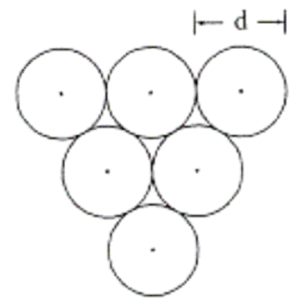

(a)

Individual

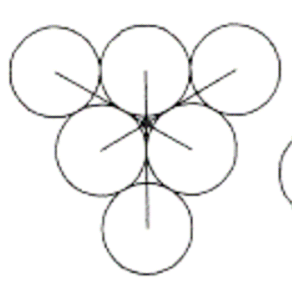

(b)

Radial

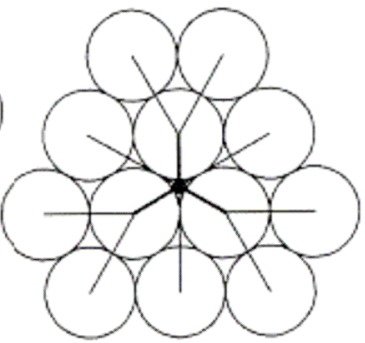

(c)

Figure 2: Distributed energy systems for heating: (a) Individual heaters; (b) central heater and radial distribution lines; (c) central heater and dendritic distribution network [1].

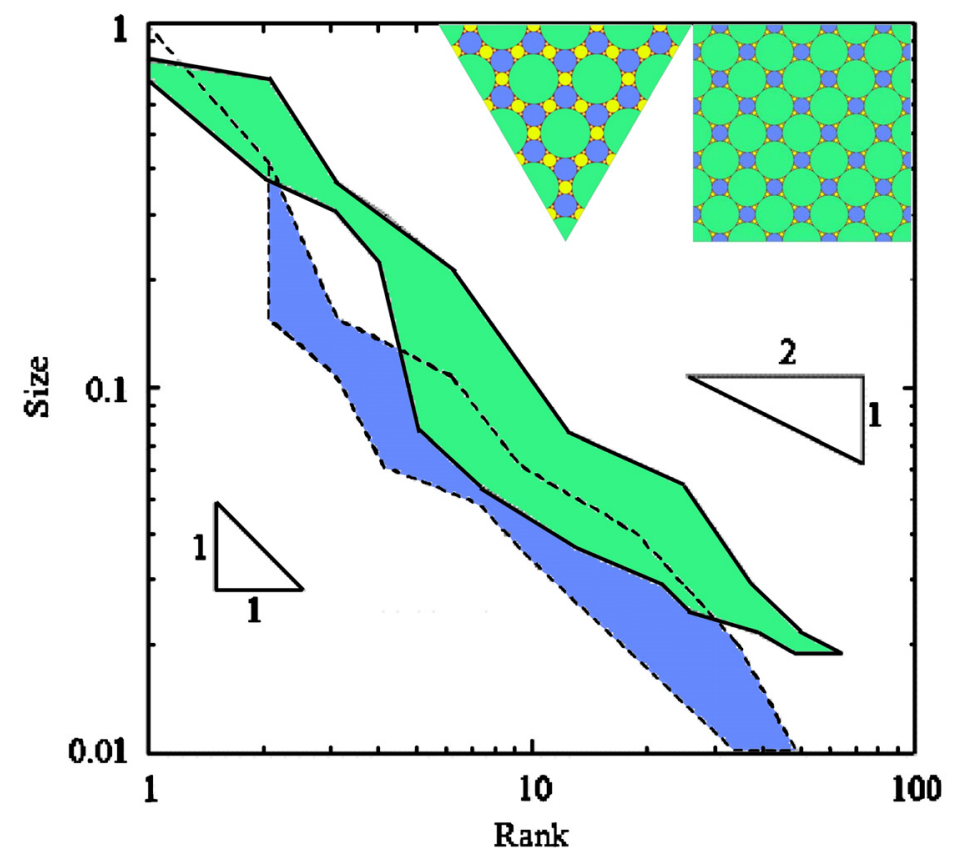

Figure 3: Distribution of canopy sizes versus rank in the constructal design of the forest floor $[1,12]$.

more complex. The landscape is covered by design, but the design changes abruptly [from (a) to (b) and (c)] as time passes. This is the essential aspect of technology evolution, and how it is anticipated based on principle.

The third domain in which we predicted the same emergence of multi-scale configuration on the landscape is the distribution of tree sizes and numbers on the forest floor $[1,12]$. With the constructal law, we deduced the configuration 
of each tree (root, trunk, canopy) as a flow system morphing to facilitate two flows: water and stresses. This led to several predictions of vegetation design (e.g., Leonardo's rule, Fibonacci sequence, Huber's rule), including the proportionality between the mass flow rate (from ground to air) through one tree and the diameter of the canopy. Next, from the tendency of the entire forest floor to transfer water to the atmosphere, came the multi-patch design of the forest floor (Fig. 3). The predicted distribution of tree sizes versus rank is again of the Zipf type, and is the second instance in which we discovered the Zipf distribution as a consequence of the constructal law.

\section{Multi-scale energy systems distributed on the landscape}

The generation and use of power as a global design is an important relative of the constructal designs reviewed in Figs. 1-3. The global energy "tapestry" is much more extensive than the power plants themselves. The tapestry contains two major parts:

(i) The power plants with their networks of power distribution, and

(ii) The areas from which fuel is harvested and the supply systems that serves the power plants.

Consider the question of how to generate more power with the fuel that is available on a territory of fixed size, such as a country or continent. Assume that the territory is divided into area elements of size A. Each area element supplies fuel for the single power plant located on A. Should A be large or small? In other words, should the territory be served by a few large power plants or by many smaller power plants?

This is an important and very basic question that finds its answer naturally in how the globe is covered by nodes of power generation in a given technological age. The allocation of nodes to areas happens "naturally" because they result from a competition between parts (i) and (ii). The efficiency of the power plant is higher when the power plant is larger $[2,13]$. In the same limit, the elemental area $\mathrm{A}$ increases and so does the fuel cost associated with harvesting and transporting fuel from A to the power plant. From this trade off emerges the equilibrium between the two parts of the flow system, (i) and (ii). This is represented by the size of A or the number of power plants (or area A) that are spread over the fixed-size territory.

Analytically, this can be demonstrated by considering the power generated by the power plant located on one elemental area A,

$$
\mathrm{W}=\eta \mathrm{Q}
$$

The area A sustains the power plant. The heat input $\mathrm{Q}$ is proportional to the rate at which fuel flows into the power plant, which in turn is proportional to A,

$$
\mathrm{Q}=\mathrm{aA}, \quad \mathrm{a}=\mathrm{constant}
$$


Next, from constructal theory $[1,4]$ we know that the power spent on moving mass on the surface of the earth is proportional to the rate at which mass is flowing times the distance traveled. In the case of the fuel collected from area A the mass flow rate is proportional to $\mathrm{A}$, and the distance scales as $\mathrm{A}^{1 / 2}$. Therefore the power required by the fuel harvesting system is $b_{A A}{ }^{1 / 2}$, where $b$ is a constant. The net power that is produced on $\mathrm{A}$ is the difference between $\mathrm{W}$ and the fuel harvesting penalty,

$$
\mathrm{W}_{\text {net }}=\mathrm{aA} \eta-\mathrm{bA}^{3 / 2}
$$

The efficiency $\eta$ increases with the capacity of the power plant (Q, or A), cf. Refs. $[2,13]$. Although rough and time-dependent, the relation is monotonic because technology evolves, and concave because $\eta$ cannot rise above 1. An adequate curve fit of such data is of the form

$$
\eta=1-m e^{-n A}
$$

In a given technological era, $\mathrm{m}$ and $\mathrm{n}$ are two constants. Efficiencies increase slowly in time, therefore $\mathrm{m}(\mathrm{t})$ decreases as technology improves.

The basic question is how much net power is produced per unit area on the given territory. This quantity is the same as $\mathrm{W}_{\text {net }} / \mathrm{A}$, and it is a function of the size of the elemental area,

$$
\frac{\mathrm{W}_{\text {net }}}{\mathrm{aA}}=\eta-\frac{\mathrm{b}}{\mathrm{a}} \mathrm{A}^{1 / 2}
$$

Figure 4 shows that this function is positive in a certain range of $A$ values. It is maximum when $A$ is such that $d\left(W_{\text {net }} / A\right) d A=0$, or if we use Eq. (4), when

$$
2 \mathrm{~A}^{1 / 2} \mathrm{e}^{-\mathrm{nA}}=\frac{\mathrm{b}}{\mathrm{amn}} \text {, constant }
$$

The finite range in which net power generation is possible means two things: the power plant size (or A) must be large enough so that the power plant is efficient, and the size must not exceed a certain limit such that losses due to transport and distribution are not prohibitive.

The focus on the generation of net power per unit of territory is similar to the approach taken in Fig. 2, where the objective was more hot water for the user (i.e. less heat loss per unit of territory). Because $\mathrm{m}(\mathrm{t})$ decreases as technology evolves, Eq. (6) predicts that A will slowly increase in time: power plants will be larger nodules, on larger areas will be allocated to each power plant.

\section{Conclusion: the future belongs to the distributed and vascularized}

In summary, the global design phenomena of Figs. 1-4 show that there is an optimal way in which to allocate an inhabited territory (the size of the area) to a 

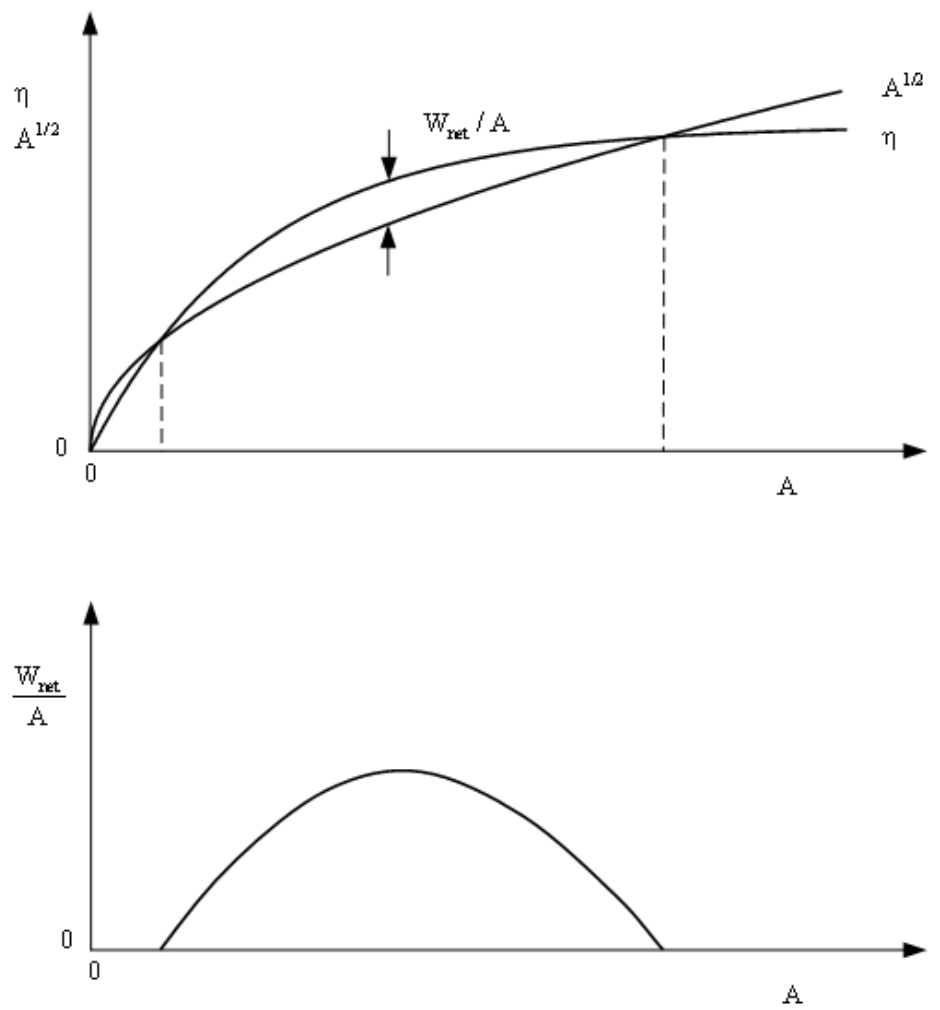

Figure 4: The effect of size (A, or Q) on the efficiency of the power plant $(\eta)$ and the length scale of the area allocated to the power plant (A1/2). The bottom figure shows the effect of size on the net power produced per unit of territory.

concentration of flow (city, heating, vegetation). Every point of the area is served best by the distributed energy system when the losses that occur in the central entity are of the same order as the losses distributed along the lines of the distribution network.

A first-time review of a fresh direction of research is a good predictor of what lies ahead on the same route. The distributed designs reviewed in this paper have this quality. The emerging field is outlined in detail as a course on how to pursue design as science, based on the constructal law [1]. Newer articles show how to use the constructal law in the development of designed multiscale media with vascular architectures [4, 15] and electrokinetic mass transfer [16], and science for green energy, security and sustainability [17-19].

\section{References}

[1] Bejan, A. and Lorente, S., Design with Constructal Theory, Wiley: Hoboken, 2008. 
[2] Bejan, A., Advanced Engineering Thermodynamics, $2^{\text {nd }}$ ed., Wiley, New York, 1997.

[3] Bejan, A., Shape and Structure, from Engineering to Nature, Cambridge University Press, Cambridge, UK, 2000.

[4] Bejan, A. and Lorente, S., Constructal theory of configuration generation in nature and engineering, J. Appl. Phys., 100, 041301, 2006.

[5] Reis, A. H., Constructal theory: from engineering to physics, and how flow systems develop shape and structure, Appl. Mech. Rev., 59, pp. 269282, 2006.

[6] Poirier, H., A theory explains the intelligence of nature. Science \& Vie, 1034, pp. 44-63, 2003.

[7] Reis, A. H., Constructal view of scaling laws of river basins, Geomorphology, 78, pp. 201-206, 2006.

[8] Constructal Theory Web Portal, www.constructal.org

[9] Miguel, A. F., Constructal pattern formation in stony corals, bacterial colonies and plant roots under different hydrodynamics conditions, $J$. Theor. Biol., 242, pp. 954-961, 2006.

[10] Bejan, A. \& Marden, J. H., Constructing animal locomotion from new thermodynamics theory, American Scientist, 94, pp. 342-349, 2006.

[11] Bejan, A., Lorente, S., Miguel, A. F. and Reis, A. H., Constructal theory of distribution of city sizes, in Bejan, A., Advanced Engineering Thermodynamics, $3^{\text {rd }}$ edn, Wiley: Hoboken, 2006.

[12] Bejan, A., Lorente, S. and Lee, J., Unifying constructal theory of tree roots, canopies and forests, J. Theor. Biol., 254(3), pp. 529-540, 2008.

[13] Kim, Y., Lorente, S. and Bejan, A., Distribution of size in steam turbine power plants, Int. J. Energy Research, 2009, to appear.

[14] Lorente, S. and Bejan, A., Heterogeneous porous media as multiscale structures for maximum flow access, Journal of Applied Physics, 100, 2006, 114909.

[15] Kim, S., Lorente, S., Bejan, A., Miller, W. and Morse, J., The emergence of vascular design in three dimensions, Journal of Applied Physics, 103, 2008, 123511.

[16] Lorente, S. and Bejan, A., Constructal design of vascular porous materials and electrokinetic mass transfer, Transport in Porous Media, 77, pp. 305322, 2009.

[17] Bejan, A. and Lorente, S., Constructal theory and its relevance to green energy, International Journal of Green Energy, 4, pp. 105-117, 2007.

[18] Bejan, A. and Merkx, G. W., eds., Constructal Theory of Social Dynamics, Springer, New York, 2007.

[19] Bejan, A., Lorente, S., Miguel, A. and Reis, A. H., eds., Constructal Human Dynamics, Security and Sustainability, IOS Press, Amsterdam, 2009. 